\title{
Household income and medical help-seeking for fertility problems among a representative population in Japan
}

\author{
Arisa Iba', Eri Maeda², Seung Chik Jwa ${ }^{3}$, Ayako Yanagisawa-Sugita', Kazuki Saito ${ }^{4}$, Akira Kuwahara ${ }^{5}$, \\ Hidekazu Saito ${ }^{6}$, Yukihiro Terada ${ }^{7}$, Osamu Ishihara ${ }^{3}$ and Yasuki Kobayashi ${ }^{*}$ (])
}

\begin{abstract}
Background: Fertility treatments help many infertile couples to have children. However, disparities exist in access to fertility tests and treatments. We investigated the association between household income and medical help-seeking for fertility in Japan.

Methods: We conducted a cross-sectional study using nationally representative data from the National Fertility Survey 2015. Respondents were 6598 married women younger than 50 years old. The primary outcome was medical help-seeking for fertility among those who experienced fertility problems. Multiple logistic regression models were used to assess the association between household income and medical help-seeking, adjusting for age, length of marriage, educational level, employment status, number of children, childbearing desires, living with parents, and region of residence.
\end{abstract}

Results: Among 2253 (34\%) women who experienced fertility problems, 1154 (51\%) sought medical help. The proportion of help-seekers increased linearly from $43 \%$ in the low-income group ( $<4$ million Japanese yen [JPY]) to 59\% in the high-income group ( $\geq 8$ million JPY) ( $P$ for trend $<0.001$ ). Respondents with upper-middle (6-8 million JPY) or high household income were more likely to seek medical help, compared to those with low household income: adjusted odds ratio [aOR] 1.37 (95\% confidence interval [Cl]: 1.00-1.86) and aOR 1.78 (95\% Cl: 1.29-2.47), respectively.

Conclusions: We found that higher household income was associated with a higher probability of seeking medical help among Japanese women who experienced fertility problem. Along with policy discussion about additional financial support, further studies from societal, cultural, or psychological views are required.

\section{Plain Language Summary}

Fertility treatments have helped millions of people to have a child. Although financial factors are known to play an important role in the decision to use fertility treatments, no previous studies have investigated how socioeconomic factors affect medical help-seeking for fertility in Japan. Therefore, we assessed the association between household income and medical help-seeking among couples with fertility problems in Japan, using nationally representative data from the National Fertility Survey 2015. Of the 6598 respondents (married women under 50 years old), onethird (2253) reported worrying about fertility problems, and half of those women (1154) sought medical help for

\footnotetext{
*Correspondence: yasukik@m.u-tokyo.ac.jp

${ }^{1}$ Department of Public Health, Graduate School of Medicine, The University of Tokyo, 7-3-1 Hongo, Bunkyo-ku, Tokyo 113-0033, Japan

Full list of author information is available at the end of the article
} permits use, sharing, adaptation, distribution and reproduction in any medium or format, as long as you give appropriate credit to the original author(s) and the source, provide a link to the Creative Commons licence, and indicate if changes were made. The images or other third party material in this article are included in the article's Creative Commons licence, unless indicated otherwise in a credit line to the material. If material is not included in the article's Creative Commons licence and your intended use is not permitted by statutory regulation or exceeds the permitted use, you will need to obtain permission directly from the copyright holder. To view a copy of this licence, visit http://creativecommons.org/licenses/by/4.0/. The Creative Commons Public Domain Dedication waiver (http://creativeco mmons.org/publicdomain/zero/1.0/) applies to the data made available in this article, unless otherwise stated in a credit line to the data. 
fertility-related issues. The proportion of help-seekers was highest (59\%) among those with high household income and lowest (43\%) among those with low household income with a significant linear trend. Even after accounting for age, length of marriage, educational level, employment status, and other possibly related factors, those with higher household income were more likely to seek medical help for fertility tests and treatments. Japan provides various sources of financial support for fertility-related care, such as health insurance coverage for tests and early-stage treatments and partial subsidies for assisted reproductive technology treatments. However, the results indicate that further policy discussion about additional financial support and further studies focusing on barriers to care in Japan could help improve the situations for those with fertility problems.

Keywords: Infertility, Care-seeking, Healthcare disparities, Socioeconomic status, Japan

\section{Background}

Infertility is a global public health issue $[1,2]$. The need for and utilization of fertility treatments is increasing [3-6] as more people delay parenthood [7-9]. Fertility treatments have helped millions of people to have a child, but disparities in access to care persist. Various factors (e.g., financial, social, demographic, or psychological) contribute to these disparities $[10,11]$. Financial accessibility plays a particularly important role in the decision to use fertility treatments $[8,12]$. Among women who report infertility, those with higher income are more likely to seek a medical evaluation for infertility [13] and to use multiple and advanced treatments $[12,14,15]$. Even in Germany and Australia, where the public healthcare systems cover fertility treatments, patients living in high-income areas use more assisted reproductive technology (ART) cycles [16, 17]. Besides income, the cost is a critical factor in access to treatment; for example, reduction in out-of-pocket expenses with mandated health insurance coverage for fertility care is associated with increased help-seeking and utilization of fertility treatments in the United States [18, 19].

In Japan, where the total fertility rate is low (1.42 in 2018) and the mean parental age at first birth is high (30.7 and 32.8 years for women and men, respectively, in 2018) [20], $18.2 \%$ of married couples receive medical examinations or treatments for infertility, according to a national survey [21]. With Japan's universal health insurance coverage [22], people can receive medical tests for fertility problem including hormonal test, hysterosalpingography, and early-phase fertility treatments including ovulation induction with timed intercourse using transvaginal ultrasonography with $30 \%$ copayments. On the other hand, intrauterine insemination and ART treatments are not covered by public health insurance. Alternatively, the government offers partial reimbursement of 300,000 Japanese Yen (JPY) (i.e., about 2,800 US dollars [USD]) per ART cycle for up to six cycles, for women younger than 43 [23], and the cost per fresh cycle in Japan is relatively low in the world [24], at approximately 400,000 JPY [25], or 3700 USD using the 2019 exchange rate of 1 USD $=109$ JPY.

Japan provides adequate financial accessibility to fertility treatments, compared to most developed countries [26]. However, given the disparities observed in countries providing public funding for fertility treatments [16, 17], financial barriers to fertility care may still exist in Japan [27]. To date, no previous study has investigated the association between socioeconomic factors and medical help-seeking for fertility in Japan. Therefore, we aimed to explore the association between household income and medical help-seeking among couples with fertility problems in Japan, using nationally representative cross-sectional data from the National Fertility Survey.

\section{Methods \\ Data source and study population}

We used data from the Married Couples Survey of the 15th National Fertility Survey, conducted in June 2015. The National Fertility Survey was carried out by the National Institute of Population and Social Security Research under the Ministry of Health, Labour, and Welfare to collect nationally representative data on topics related to marriage, childbirth, and child-rearing in Japan since 1977 [21, 28]. The survey used a stratified, random cluster sampling method to select $900 \mathrm{enu}$ meration districts from the 2010 Population Census [28]. Eligible candidates for the Married Couples Survey were married women younger than 50 years old who lived in these 900 districts, including foreign residents who spoke Japanese [28]. Eligible people received a self-administered questionnaire through home visits, and upon completion, returned it in a sealed envelope at a follow-up visit [21, 29]. Respondents provided information about their husbands and themselves. The number of valid responses was 6598 (87.8\% collection rate) [21]. Data on primary sampling units and stratification were not available due to constraints on secondary data usage. 


\section{Fertility status and medical help-seeking}

Participants were asked whether they had ever worried about fertility problems. We categorized as subfertile those who reported current or previous subfertility by choosing the answer, "We (i.e., my husband and I) are currently worried about not being able to have a child" or "We were worried about not being able to have a child in the past." We categorized as fertile those participants who chose the answer, "We have never worried about not being able to have a child." Since the National Fertility Survey asked only about couples' fertility concerns, not infertility by medical definition [2], we defined fertile and subfertile according to respondents' perceptions of their relative fertility.

Subfertile participants were asked whether they had ever sought medical help for fertility problems. Those who chose the answer "We have never sought medical help" were categorized as non-help-seekers. Those who chose "We are currently undergoing testing or treatment" or "We have been tested or treated in the past" were categorized as help-seekers.

\section{Household income and other possible related factors}

The participants reported their own and their spouse's annual incomes from the previous year in increments of one million JPY (e.g., $\geq 2$ million JPY and $<3$ million JPY). We estimated annual household income by summing the midpoint value of each increment (e.g., 2.5 million JPY) for both participant and husband and then categorized income into four groups: low $(<4$ million JPY), lowermiddle ( $\geq 4$ million JPY to $<6$ million JPY), upper-middle ( $\geq 6$ million JPY to $<8$ million JPY), and high ( $\geq 8$ million JPY).

We used the following sociodemographic variables as possible factors related to help-seeking: age (i.e., $\leq 29$, $30-34,35-39, \geq 40$ ), educational level (i.e., high school education or less, vocational or junior college education, university education or higher), employment status of participant and spouse at the time of the survey (i.e., full-time, part-time, self-employed, unemployed), length of marriage (i.e., $\leq 4$ years, 5-9 years, $10-14$ years, $\geq 15$ years), number of existing children at the time of the survey $(0,1,2$, or $\geq 3)$, desire to have one or more children at the time of the survey (yes/no), living with their parents after marriage (yes/no), residential region (i.e., seven regions of Japan), and population size and density of the residential district (i.e., non-densely inhabited, less than 200,000 inhabitants, 200,000 to $1,000,000$ inhabitants, more than 1,000,000 inhabitants) [30]. We gave an affirmative score to the desire to have additional children at the time of the survey if participants' ideal number of children exceeded their reported number of children at that time. No questions were asked about respondents' ethnicity, race or nationality, since the proportion of foreign residents in Japan is low (1.4\% as of 2015) [31] and ethnicity questions are sensitive to some Japanese.

\section{Statistical analysis}

We described the distributions of sociodemographic variables according to fertility status. Chi-square tests were used to compare the proportions of nominal variables (i.e., employment status, desire to have a child, living with parents, and region of residence), and a Wilcoxontype test for trend was used to compare the proportions of ordinal variables (e.g., age and length of marriage) between fertile and subfertile groups.

The primary outcome was medical help-seeking among those who experienced fertility problems. Thus, we conducted the following analyses using the subfertile subgroup. We described the distribution of the variables according to help-seeking behavior and then used chisquare tests to compare the proportion of nominal variables and Wilcoxon-type tests for trend to compare the proportion of ordinal variables between help-seekers and non-help-seekers. Univariable logistic regression was used to explore the association of each variable with help-seeking.

We conducted multiple logistic regression analyses to assess the association between household income and medical help-seeking, controlling for the other covariates. We excluded the husband's age from the multivariable model due to multicollinearity with the wife's age. Although the proportion of missing values was small $(<6 \%)$ for all variables, $386(17 \%)$ subfertile respondents had missing data for one or more investigated variable. Thus, in addition to the complete case analysis, we conducted multiple imputations for the multivariable regression analysis using the multivariate normal imputation method to account for missing data. We included all explanatory variables and the outcome variable [32] (1.2\% missingness) in the imputation model, creating 20 multiply imputed data sets. Categorical variables were imputed using a set of binary indicators for each category, and unrounded values were used for the analysis [33].

We also conducted sensitivity analyses using data of respondents who reported current subfertility to analyze whether the association of household income and helpseeking at the time when they faced fertility problems is consistent. We omitted variables regarding husbands' age, educational level, and employment status, living with parents, and residential regions to prevent overfitting. A two-sided $P$ value of $<0.05$ was used to define statistical 
significance. All analyses were performed using Stata version 14.2 (StataCorp., College Station, TX, USA).

\section{Ethical considerations}

The study protocol was approved by the institutional review board of the Graduate School of Medicine at The University of Tokyo (approval no: 2019270NI, approved on January 23, 2020) and by the ethics committee of Akita University Graduate School of Medicine (approval no: 2300, approved on September 20, 2019). The Ministry of Health, Labor and Welfare approved the secondary use of data from the National Fertility Survey, and informed consent was not required because of anonymous data.

\section{Results}

Of the 6598 valid respondents (i.e., wives), 341 (5\%) and 1912 (29\%) reported current and previous fertility problems, respectively. Thus, 2253 (34\%) respondents were categorized as subfertile (Table 1). Wives and husbands of the subfertile group were significantly more educated and had longer marriages, higher household incomes, and fewer children at the time of the survey than those of the fertile group (all $P_{\mathbf{S}}<0.05$ ). The proportion of those desiring one or more child at the time of the survey was significantly higher in the subfertile group (68\%) than in the fertile group $(42 \%, P<0.001)$.

Among subfertile respondents, 1154 (51\%) sought medical help for fertility problems (Table 1 ). Compared to non-help-seekers, medical help-seekers were significantly older and more educated, with longer marriages and higher household incomes, and they lived in more densely-inhabited districts (all $P \mathrm{~s}<0.05$ ). The proportions of medical help-seekers in each income group were $43 \%$ in the low-, $50 \%$ in the lower-middle-, $52 \%$ in the upper-middle-, and $59 \%$ in the high-income groups with significant linear trend $(P$ for trend $<0.001)$, respectively (Fig. 1). The proportions of those desiring a child at the time of the survey were similar between medical helpseekers and non-help-seekers.

Table 2 shows the results of the univariable and multivariable logistic regression analyses for medical help-seeking. In the univariable analyses, those with upper-middle or high household income were more likely to seek medical help for fertility problems, compared to those with low household income among subfertile respondents: odds ratio (OR) 1.44 (95\% confidence interval [CI] 1.09-1.90) and OR 1.90 (95\% CI 1.46-2.48), respectively. Also, older ages of wives and husbands, longer marriages, and higher education levels of wives and husbands were significantly associated with helpseeking for fertility problems.

In the multivariable analysis using multiple imputations, participants with upper-middle or high household income were more likely to seek help for fertility problems, compared to participants with low household income, after adjusting for the covariates: adjusted OR (aOR) 1.37 (95\% CI 1.00-1.86) and aOR 1.78 (95\% CI 1.29-2.47), respectively. Longer marriages, husbands having university education, and unemployment of wives were positively associated with help-seeking. Participants who had two or more children were less likely to seek medical help for fertility problems than those who had no child at the time of the survey. A complete case analysis showed similar results to that of the multiple imputation methods.

Additional file 1: Table S1 shows distribution of sociodemographic factors among those who reported current subfertility. Multiple logistic regression analyses showed positive associations between household income and help-seeking among those with current subfertility, although the results were not statistically significant (Additional file 1: Table S2): aORs for upper-middle and high household incomes were 1.47 (95\% CI 0.68-3.16) and 1.58 (95\% CI $0.72-3.46)$, respectively.

\section{Discussion}

Using a nationally representative survey, we investigated the association between household income and medical help-seeking among couples who experienced fertility problems. Approximately one third of participants reported fertility problems, for which half sought medical help. Couples with higher household income were more likely to seek medical help than those with lower household income. This study is the first to present the association between socioeconomic factors and help-seeking for fertility in Japan.

Among couples with fertility problems, 51\% sought medical help. The proportion of help-seekers was similar or slightly lower than the international estimate of $56 \%$ reported by a previous systematic review [1] and by recent studies showing $57 \%$ in the United Kingdom [11] and 55\% in China [34]. Among presumably healthconscious participants of the Nurses' Health Study II in the United States, the proportion of those receiving medical evaluations for infertility was $65 \%$, varying from $59 \%$ in the low-income group to $69 \%$ in the high-income group [13]. The National Fertility Survey in the current study asked participants to report their fertility concerns but not the experience of infertility, defined as "failing to achieve pregnancy after at least 12 months of unprotected regular sexual intercourse" [2]. Thus, the present proportion of help-seekers would not be comparable to previous works. Nonetheless, it remains a concern that a substantial proportion of couples did not seek medical help for their fertility problems. 
Table 1 Sociodemographic characteristics of participants according to fertility status and medical help-seeking

\begin{tabular}{|c|c|c|c|c|c|c|c|c|c|c|}
\hline & \multirow{2}{*}{\multicolumn{2}{|c|}{ Fertile $(n=3812)$}} & \multirow{2}{*}{\multicolumn{2}{|c|}{$\begin{array}{l}\text { Subfertile } \\
(n=2253)\end{array}$}} & \multirow[t]{3}{*}{$p^{a}$} & \multicolumn{4}{|c|}{ Subfertile (aforementioned) } & \multirow[t]{3}{*}{$p^{a}$} \\
\hline & & & & & & \multicolumn{2}{|c|}{$\begin{array}{l}\text { Non-help- } \\
\text { seekers } \\
(\mathrm{n}=1071)\end{array}$} & \multicolumn{2}{|c|}{$\begin{array}{l}\text { Help-seekers } \\
(n=1154)\end{array}$} & \\
\hline & $\mathrm{n}$ & $\%$ & $\mathrm{n}$ & $\%$ & & $\mathrm{n}$ & $\%$ & $\mathrm{n}$ & $\%$ & \\
\hline \multicolumn{11}{|l|}{ Wife's age, years } \\
\hline$\leq 29$ & 310 & 8.1 & 135 & 6.0 & 0.80 & 87 & 8.1 & 47 & 4.1 & $<0.001$ \\
\hline $30-34$ & 516 & 14 & 339 & 15 & & 183 & 17 & 151 & 13 & \\
\hline $35-39$ & 763 & 20 & 542 & 24 & & 272 & 25 & 264 & 23 & \\
\hline$\geq 40$ & 2223 & 58 & 1237 & 55 & & 529 & 49 & 692 & 60 & \\
\hline \multicolumn{11}{|l|}{ Husband's age, years } \\
\hline$\leq 29$ & 210 & 5.6 & 95 & 4.2 & 0.40 & 63 & 5.9 & 30 & 2.6 & $<0.001$ \\
\hline $30-34$ & 446 & 12 & 290 & 13 & & 173 & 16 & 114 & 10 & \\
\hline $35-39$ & 621 & 16 & 447 & 20 & & 227 & 21 & 215 & 19 & \\
\hline$\geq 40$ & 2504 & 66 & 1,404 & 63 & & 599 & 56 & 787 & 69 & \\
\hline \multicolumn{11}{|l|}{ Length of the marriage, years } \\
\hline$\leq 4$ & 546 & 15 & 415 & 19 & $<0.001$ & 248 & 24 & 161 & 14 & $<0.001$ \\
\hline $5-9$ & 703 & 19 & 557 & 25 & & 275 & 26 & 277 & 25 & \\
\hline $10-14$ & 760 & 20 & 512 & 23 & & 219 & 21 & 287 & 25 & \\
\hline$\geq 15$ & 1708 & 46 & 720 & 33 & & 305 & 29 & 405 & 36 & \\
\hline \multicolumn{11}{|l|}{ Wife's educational level } \\
\hline High school education or less & 1586 & 42 & 694 & 31 & $<0.001$ & 359 & 34 & 326 & 28 & 0.01 \\
\hline Vocational or junior college education & 1473 & 39 & 985 & 44 & & 452 & 42 & 520 & 45 & \\
\hline University education or higher & 747 & 20 & 568 & 25 & & 257 & 24 & 305 & 26 & \\
\hline Other & 5 & 0.1 & 2 & 0.1 & & 0 & 0.0 & 2 & 0.2 & \\
\hline \multicolumn{11}{|l|}{ Husband's educational level } \\
\hline High school education or less & 1659 & 44 & 788 & 35 & $<0.001$ & 416 & 39 & 362 & 31 & $<0.001$ \\
\hline Vocational or junior college education & 670 & 18 & 428 & 19 & & 201 & 19 & 223 & 19 & \\
\hline University education or higher & 1451 & 38 & 1025 & 46 & & 446 & 42 & 565 & 49 & \\
\hline Other & 9 & 0.2 & 2 & 0.1 & & 0 & 0.0 & 2 & 0.2 & \\
\hline \multicolumn{11}{|l|}{ Wife's employment status } \\
\hline Full-time worker & 849 & 23 & 577 & 26 & $<0.001$ & 283 & 27 & 286 & 25 & 0.78 \\
\hline Part-time worker & 1543 & 41 & 772 & 35 & & 361 & 34 & 400 & 35 & \\
\hline Self-employed & 222 & 5.9 & 119 & 5.4 & & 58 & 5.5 & 61 & 5.3 & \\
\hline Unemployed & 1125 & 30 & 752 & 34 & & 350 & 33 & 394 & 35 & \\
\hline \multicolumn{11}{|l|}{ Husband's employment status } \\
\hline Full-time worker & 2829 & 79 & 1,739 & 81 & 0.20 & 815 & 81 & 905 & 82 & 0.90 \\
\hline Part-time worker & 241 & 6.7 & 137 & 6.4 & & 69 & 6.9 & 67 & 6.0 & \\
\hline Self-employed & 448 & 13 & 235 & 11 & & 110 & 11 & 122 & 11 & \\
\hline Unemployed & 57 & 1.6 & 27 & 1.3 & & 13 & 1.3 & 14 & 1.3 & \\
\hline \multicolumn{11}{|l|}{ Household income ${ }^{b}$} \\
\hline Low & 659 & 18 & 330 & 16 & 0.001 & 186 & 19 & 139 & 13 & $<0.001$ \\
\hline Lower-middle & 945 & 26 & 527 & 25 & & 263 & 26 & 258 & 24 & \\
\hline Upper-middle & 844 & 23 & 519 & 24 & & 249 & 25 & 268 & 24 & \\
\hline High & 1145 & 32 & 743 & 35 & & 302 & 30 & 429 & 39 & \\
\hline \multicolumn{11}{|l|}{ Number of existing children } \\
\hline 0 & 404 & 11 & 507 & 23 & $<0.001$ & 241 & 23 & 259 & 23 & 0.06 \\
\hline 1 & 832 & 22 & 738 & 33 & & 326 & 31 & 404 & 35 & \\
\hline 2 & 1812 & 48 & 785 & 35 & & 381 & 36 & 394 & 34 & \\
\hline$\geq 3$ & 740 & 20 & 211 & 9.4 & & 118 & 11 & 92 & 8.0 & \\
\hline
\end{tabular}


Table 1 (continued)

\begin{tabular}{|c|c|c|c|c|c|c|c|c|c|c|}
\hline & \multirow{2}{*}{\multicolumn{2}{|c|}{ Fertile $(n=3812)$}} & \multirow{2}{*}{\multicolumn{2}{|c|}{$\begin{array}{l}\text { Subfertile } \\
(n=2253)\end{array}$}} & \multirow[t]{3}{*}{$\mathrm{p}^{\mathrm{a}}$} & \multicolumn{4}{|c|}{ Subfertile (aforementioned) } & \multirow[t]{3}{*}{$p^{a}$} \\
\hline & & & & & & \multicolumn{2}{|c|}{$\begin{array}{l}\begin{array}{l}\text { Non-help- } \\
\text { seekers }\end{array} \\
(\mathrm{n}=1071)\end{array}$} & \multicolumn{2}{|c|}{$\begin{array}{l}\text { Help-seekers } \\
(n=1154)\end{array}$} & \\
\hline & $\mathbf{n}$ & $\%$ & $\mathbf{n}$ & $\%$ & & $\mathbf{n}$ & $\%$ & $\mathrm{n}$ & $\%$ & \\
\hline \multicolumn{11}{|l|}{ Living with parents } \\
\hline Yes & 1076 & 29 & 562 & 25 & \multirow[t]{2}{*}{0.004} & 270 & 26 & 283 & 25 & \multirow[t]{2}{*}{0.80} \\
\hline No & 2639 & 71 & 1645 & 75 & & 785 & 74 & 844 & 75 & \\
\hline \multicolumn{11}{|l|}{ Desire to have a child } \\
\hline Yes & 1552 & 42 & 1503 & 68 & \multirow[t]{2}{*}{$<0.001$} & 709 & 68 & 780 & 69 & \multirow[t]{2}{*}{0.51} \\
\hline No & 2170 & 58 & 705 & 32 & & 341 & 32 & 353 & 31 & \\
\hline \multicolumn{11}{|l|}{ Residential region } \\
\hline Hokkaido & 129 & 3.4 & 60 & 2.7 & \multirow[t]{7}{*}{0.27} & 31 & 2.9 & 27 & 2.3 & \multirow[t]{7}{*}{0.44} \\
\hline Tohoku & 237 & 6.2 & 148 & 6.6 & & 79 & 7.4 & 68 & 5.9 & \\
\hline Kanto & 1262 & 33 & 776 & 34 & & 375 & 35 & 393 & 34 & \\
\hline Chubu & 794 & 21 & 480 & 21 & & 231 & 22 & 244 & 21 & \\
\hline Kinki & 625 & 16 & 354 & 16 & & 154 & 14 & 195 & 17 & \\
\hline Chugoku/Shikoku & 351 & 9.2 & 223 & 10 & & 99 & 9.2 & 121 & 10 & \\
\hline Kyushu/Okinawa & 414 & 11 & 212 & 9.4 & & 102 & 10 & 106 & 9.2 & \\
\hline \multicolumn{11}{|l|}{ Population size and density } \\
\hline Non-densely inhabited district & 1089 & 29 & 591 & 26 & \multirow[t]{4}{*}{0.06} & 298 & 28 & 287 & 25 & \multirow[t]{4}{*}{0.01} \\
\hline$<200,000$ inhabitants & 909 & 24 & 555 & 25 & & 286 & 27 & 260 & 23 & \\
\hline 200,000 to $1,000,000$ inhabitants & 1089 & 29 & 643 & 29 & & 279 & 26 & 358 & 31 & \\
\hline$>1,000,000$ inhabitants & 725 & 19 & 464 & 21 & & 208 & 19 & 249 & 22 & \\
\hline
\end{tabular}

JPY Japanese Yen

${ }^{\text {a }}$ Chi-squared test for nominal variables and Wilcoxon-type test for trend for ordinal variables

${ }^{b}$ Categorized into four groups: low ( $<4$ million JPY), lower-middle ( $\geq 4$ million JPY to $<6$ million JPY), upper-middle ( $\geq 6$ million JPY to $<8$ million JPY), and high ( $\geq 8$ million JPY)

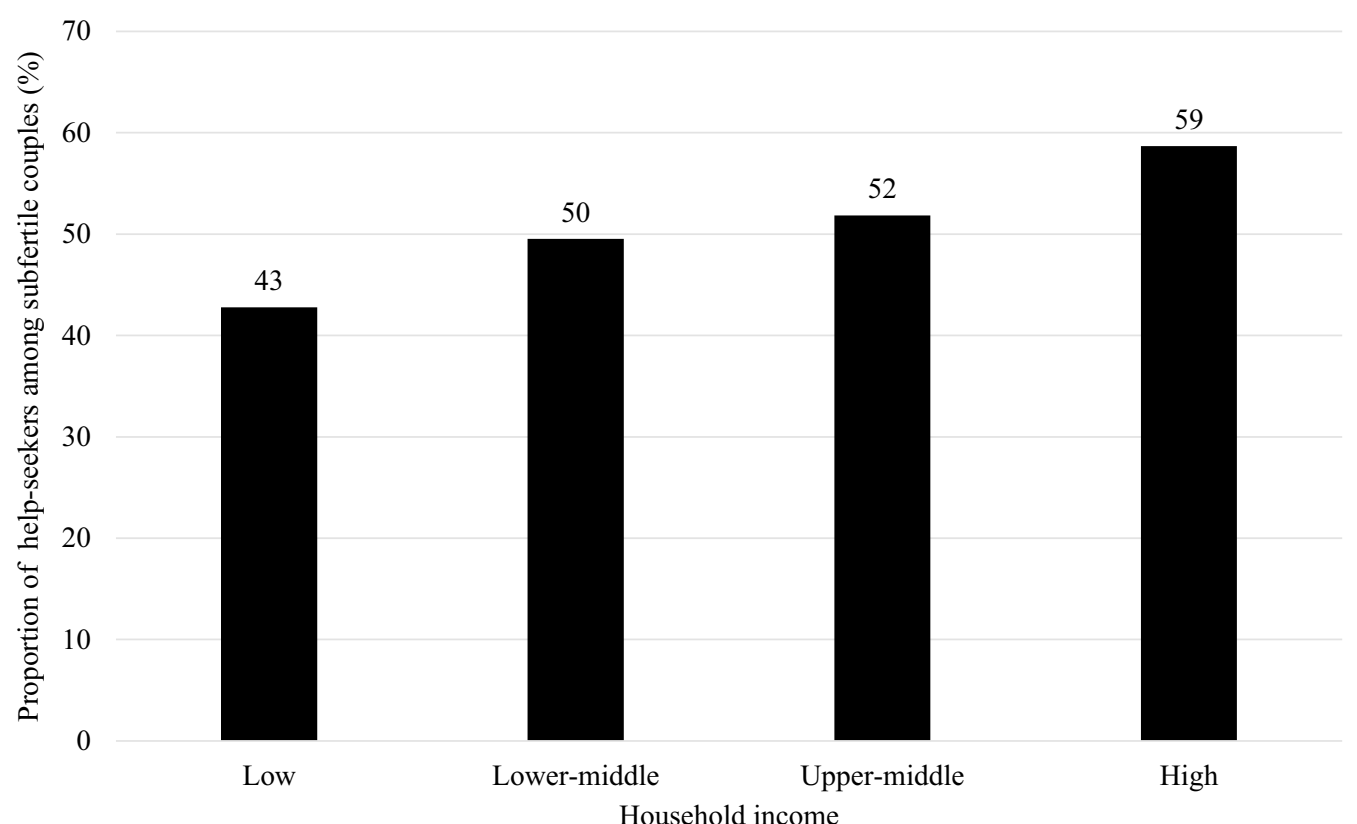

Fig. 1 Proportions of medical help-seekers for fertility problems among subfertile couples in each income group 
Table 2 Logistic regression analyses for factors associated with medical help-seeking

\begin{tabular}{|c|c|c|c|c|c|c|c|c|c|}
\hline & \multicolumn{3}{|c|}{ Univariable $(n=2225)$} & \multicolumn{3}{|c|}{$\begin{array}{l}\text { Multivariable (complete case analysis) } \\
(n=1867)\end{array}$} & \multicolumn{3}{|c|}{$\begin{array}{l}\text { Multivariable (multiple imputation) } \\
(\mathrm{n}=2253)\end{array}$} \\
\hline & Odds ratio & $95 \% \mathrm{Cl}$ & $p$ & Odds ratio & $95 \% \mathrm{Cl}$ & $\mathrm{p}$ & Odds ratio & $95 \% \mathrm{Cl}$ & $\mathrm{p}$ \\
\hline \multicolumn{10}{|c|}{ Household income ${ }^{a}$} \\
\hline Low & Ref & & & Ref & & & Ref & & \\
\hline $\begin{array}{l}\text { Lower- } \\
\text { middle }\end{array}$ & 1.31 & $0.99-1.73$ & 0.06 & 1.30 & $0.92-1.83$ & 0.14 & 1.28 & $0.95-1.73$ & 0.10 \\
\hline $\begin{array}{l}\text { Upper- } \\
\text { middle }\end{array}$ & 1.44 & $1.09-1.90$ & 0.01 & 1.44 & $1.01-2.06$ & 0.04 & 1.37 & $1.00-1.86$ & 0.05 \\
\hline High & 1.90 & $1.46-2.48$ & $<0.001$ & 1.92 & $1.32-2.78$ & 0.001 & 1.78 & $1.29-2.47$ & $<0.001$ \\
\hline \multicolumn{10}{|c|}{ Wife's age, years } \\
\hline$\leq 29$ & Ref & & & Ref & & & Ref & & \\
\hline $30-34$ & 1.53 & $1.01-2.31$ & 0.05 & 1.15 & $0.71-1.86$ & 0.58 & 1.15 & $0.74-1.79$ & 0.52 \\
\hline $35-39$ & 1.80 & $1.21-2.66$ & 0.003 & 1.14 & $0.71-1.86$ & 0.59 & 1.16 & $0.74-1.79$ & 0.52 \\
\hline$\geq 40$ & 2.42 & $1.67-3.51$ & $<0.001$ & 1.22 & $0.74-2.01$ & 0.45 & 1.28 & $0.81-2.02$ & 0.29 \\
\hline \multicolumn{10}{|c|}{ Husband's age, years } \\
\hline$\leq 29$ & Ref & & & - & - & - & - & - & - \\
\hline $30-34$ & 1.38 & $0.84-2.27$ & 0.20 & - & - & - & - & - & - \\
\hline $35-39$ & 1.99 & $1.24-3.19$ & 0.004 & - & - & - & - & - & - \\
\hline$\geq 40$ & 2.76 & $1.76-4.32$ & $<0.001$ & - & - & - & - & - & - \\
\hline \multicolumn{10}{|c|}{ Length of the marriage, years } \\
\hline$\leq 4$ & Ref & & & Ref & & & Ref & & \\
\hline $5-9$ & 1.55 & $1.20-2.01$ & 0.001 & 1.85 & $1.34-2.55$ & $<0.001$ & 1.74 & $1.30-2.33$ & $<0.001$ \\
\hline $10-14$ & 2.02 & $1.55-2.63$ & $<0.001$ & 2.33 & $1.61-3.38$ & $<0.001$ & 2.30 & $1.63-3.23$ & $<0.001$ \\
\hline$\geq 15$ & 2.05 & $1.60-2.62$ & $<0.001$ & 2.42 & $1.61-3.64$ & $<0.001$ & 2.47 & $1.70-3.59$ & $<0.001$ \\
\hline \multicolumn{10}{|c|}{ Wife's educational level } \\
\hline $\begin{array}{l}\text { High school } \\
\text { education } \\
\text { or less }\end{array}$ & Ref & & & Ref & & & Ref & & \\
\hline $\begin{array}{l}\text { Vocational } \\
\text { or junior } \\
\text { college } \\
\text { education }\end{array}$ & 1.27 & $1.04-1.54$ & 0.02 & 1.13 & $0.89-1.43$ & 0.32 & 1.11 & $0.90-1.38$ & 0.32 \\
\hline $\begin{array}{l}\text { University } \\
\text { education } \\
\text { or higher }\end{array}$ & 1.31 & $1.05-1.63$ & 0.02 & 1.11 & $0.83-1.48$ & 0.48 & 1.16 & $0.89-1.51$ & 0.27 \\
\hline \multicolumn{10}{|c|}{ Husband's educational level } \\
\hline $\begin{array}{l}\text { High school } \\
\text { education } \\
\text { or less }\end{array}$ & Ref & & & Ref & & & Ref & & \\
\hline $\begin{array}{l}\text { Vocational } \\
\text { or junior } \\
\text { college } \\
\text { education }\end{array}$ & 1.27 & $1.01-1.62$ & 0.05 & 1.28 & $0.98-1.69$ & 0.07 & 1.25 & $0.97-1.61$ & 0.08 \\
\hline $\begin{array}{l}\text { University } \\
\text { education } \\
\text { or higher }\end{array}$ & 1.46 & $1.21-1.76$ & $<0.001$ & 1.22 & $0.96-1.56$ & 0.10 & 1.27 & $1.02-1.59$ & 0.03 \\
\hline \multicolumn{10}{|c|}{ Wife's employment status } \\
\hline $\begin{array}{l}\text { Full-time } \\
\text { worker }\end{array}$ & Ref & & & Ref & & & Ref & & \\
\hline $\begin{array}{l}\text { Part-time } \\
\text { worker }\end{array}$ & 1.10 & $0.88-1.36$ & 0.41 & 1.28 & $0.98-1.69$ & 0.07 & 1.23 & $0.96-1.59$ & 0.10 \\
\hline $\begin{array}{l}\text { Self- } \\
\text { employed }\end{array}$ & 1.04 & $0.70-1.55$ & 0.84 & 1.14 & $0.71-1.83$ & 0.60 & 1.03 & $0.66-1.60$ & 0.89 \\
\hline
\end{tabular}


Table 2 (continued)

\begin{tabular}{|c|c|c|c|c|c|c|c|c|c|}
\hline & \multicolumn{3}{|c|}{ Univariable $(n=2225)$} & \multicolumn{3}{|c|}{$\begin{array}{l}\text { Multivariable (complete case analysis) } \\
(n=1867)\end{array}$} & \multicolumn{3}{|c|}{$\begin{array}{l}\text { Multivariable (multiple imputation) } \\
(n=2253)\end{array}$} \\
\hline & Odds ratio & $95 \% \mathrm{Cl}$ & $\mathrm{p}$ & Odds ratio & $95 \% \mathrm{Cl}$ & $p$ & Odds ratio & $95 \% \mathrm{Cl}$ & $\mathbf{p}$ \\
\hline $\begin{array}{l}\text { Unem- } \\
\text { ployed }\end{array}$ & 1.11 & $0.90-1.39$ & 0.33 & 1.48 & $1.12-1.95$ & 0.01 & 1.39 & $1.08-1.79$ & 0.01 \\
\hline \multicolumn{10}{|c|}{ Husband's employment status } \\
\hline $\begin{array}{l}\text { Full-time } \\
\text { worker }\end{array}$ & Ref & & & Ref & & & Ref & & \\
\hline $\begin{array}{l}\text { Part-time } \\
\text { worker }\end{array}$ & 0.87 & $0.62-1.24$ & 0.45 & 0.79 & $0.53-1.18$ & 0.24 & 0.85 & $0.58-1.25$ & 0.41 \\
\hline $\begin{array}{l}\text { Self- } \\
\text { employed }\end{array}$ & 1.00 & $0.76-1.31$ & 0.99 & 0.98 & $0.70-1.38$ & 0.92 & 1.10 & $0.80-1.50$ & 0.55 \\
\hline $\begin{array}{l}\text { Unem- } \\
\text { ployed }\end{array}$ & 0.97 & $0.45-2.08$ & 0.94 & 1.11 & $0.47-2.63$ & 0.82 & 1.33 & $0.59-3.00$ & 0.49 \\
\hline \multicolumn{10}{|c|}{ Number of existing children } \\
\hline 0 & Ref & & & Ref & & & Ref & & \\
\hline 1 & 1.15 & $0.92-1.45$ & 0.22 & 1.02 & $0.78-1.32$ & 0.90 & 0.96 & $0.75-1.22$ & 0.73 \\
\hline 2 & 0.96 & $0.77-1.20$ & 0.74 & 0.62 & $0.46-0.84$ & 0.002 & 0.62 & $0.47-0.82$ & 0.001 \\
\hline$\geq 3$ & 0.73 & $0.52-1.00$ & 0.05 & 0.48 & $0.31-0.74$ & 0.001 & 0.46 & $0.31-0.67$ & $<0.001$ \\
\hline \multicolumn{10}{|c|}{ Living with parents } \\
\hline No & Ref & & & Ref & & & Ref & & \\
\hline Yes & 0.97 & $0.80-1.18$ & 0.80 & 1.13 & $0.90-1.42$ & 0.30 & 1.11 & $0.90-1.37$ & 0.32 \\
\hline \multicolumn{10}{|c|}{ Desire to have a child } \\
\hline No & Ref & & & Ref & & & Ref & & \\
\hline Yes & 1.06 & $0.89-1.27$ & 0.51 & 1.01 & $0.78-1.31$ & 0.92 & 0.98 & $0.78-1.23$ & 0.85 \\
\hline \multicolumn{10}{|c|}{ Residential region } \\
\hline Hokkaido & Ref & & & Ref & & & Ref & & \\
\hline Tohoku & 0.99 & $0.54-1.82$ & 0.97 & 0.70 & $0.34-1.43$ & 0.32 & 0.83 & $0.45-1.56$ & 0.57 \\
\hline Kanto & 1.20 & $0.70-2.05$ & 0.50 & 0.69 & $0.36-1.32$ & 0.26 & 0.78 & $0.45-1.36$ & 0.38 \\
\hline Chubu & 1.21 & $0.70-2.09$ & 0.49 & 0.82 & $0.43-1.58$ & 0.55 & 0.88 & $0.50-1.54$ & 0.65 \\
\hline Kinki & 1.45 & $0.83-2.54$ & 0.19 & 0.92 & $0.47-1.78$ & 0.80 & 1.02 & $0.58-1.81$ & 0.95 \\
\hline $\begin{array}{l}\text { Chugoku/ } \\
\text { Shikoku }\end{array}$ & 1.40 & $0.79-2.51$ & 0.25 & 0.94 & $0.47-1.87$ & 0.86 & 1.12 & $0.62-2.03$ & 0.72 \\
\hline $\begin{array}{l}\text { Kyushu/ } \\
\text { Okinawa }\end{array}$ & 1.19 & $0.67-2.14$ & 0.55 & 0.88 & $0.44-1.76$ & 0.72 & 1.05 & $0.58-1.91$ & 0.87 \\
\hline \multicolumn{10}{|c|}{ Population size and density } \\
\hline $\begin{array}{l}\text { Non- } \\
\text { densely } \\
\text { inhabited } \\
\text { district }\end{array}$ & Ref & & & Ref & & & Ref & & \\
\hline $\begin{array}{l}<200,000 \\
\text { inhabit- } \\
\text { ants }\end{array}$ & 0.94 & $0.75-1.19$ & 0.63 & 0.93 & $0.70-1.22$ & 0.58 & 0.95 & $0.74-1.22$ & 0.68 \\
\hline $\begin{array}{l}200,000 \text { to } \\
1,000,000 \\
\text { inhabit- } \\
\text { ants }\end{array}$ & 1.33 & $1.06-1.67$ & 0.01 & 1.18 & $0.91-1.54$ & 0.21 & 1.18 & $0.93-1.50$ & 0.18 \\
\hline $\begin{array}{l}>1,000,000 \\
\text { inhabit- } \\
\text { ants }\end{array}$ & 1.24 & $0.97-1.59$ & 0.08 & 1.07 & $0.79-1.44$ & 0.68 & 1.09 & $0.83-1.43$ & 0.56 \\
\hline
\end{tabular}

$\mathrm{Cl}$ confidence interval, JPY Japanese Yen, ref reference

${ }^{\text {a }}$ Categorized into four groups: low $(<4$ million JPY), lower-middle ( $\geq 4$ million JPY to $<6$ million JPY), upper-middle ( $\geq 6$ million JPY to $<8$ million JPY), and high ( $\geq 8$ million JPY) 
The proportion of help-seekers linearly increased with household income, from $43 \%$ in the low-income group to $59 \%$ in the high-income group. The positive association between household income and medical help-seeking was consistent with previous studies conducted in other countries [12, 13, 35], even though Japan provides public health insurance coverage for fertility tests and early-phase treatments, as well as partial subsidies for ART treatments. A possible reason for this finding is that out-of-pocket payment remains expensive, relative to disposable income. For example, hysterosalpingography as a diagnostic testing for tubal factor infertility usually requires approximately 10,000 JPY (i.e., 90 USD) of copayment after health insurance coverage. Such out-of-pocket payments, as well as opportunity costs lost through medical consultations, might affect helpseeking, especially among lower-income groups. Some local governments provide subsidies for fertility tests and early-phase fertility treatments such as ovulation induction (e.g., up to 50,000 JPY in Tokyo Prefecture [36]), and some add local subsidies for ART treatments (e.g., additional 300,000 JPY per fiscal year [37] or additional three ART cycles [38]) to the national subsidy. As a future research direction, it would be necessary to evaluate the effect of such additional local subsidies on the improvements of medical help-seeking behavior among low-income group. Concerns about job security, housing security, and the cost of childcare and education may impose additional, indirect impacts on planned fertility [9]. These additional pressures are likely to compound the direct effects of income on access to fertility care, especially among those with lower household incomes.

We showed positive associations between husbands' educational level and medical help-seeking, although wives' education was not significant in the multivariable model (Table 2). Higher education is known to be associated with help-seeking behavior [11, 39]. Several explanations have been suggested from a non-economic perspective. Fertility knowledge [40] and awareness of fertility problems [41] may promote intentions to improve fertility [42] and increase access to health care [12]. Whereas help-seekers had positive treatment beliefs, such as perceived high success rates or ease of obtaining help, non-help-seekers may have more fear of discovering a problem and of being labeled infertile, as well as perceived high treatment costs. A recent localgovernment survey conducted in Japan revealed men's fertility awareness to be lower than that of their wives, and some couples failed to pursue fertility care due to the husbands' indifference [43]. A previous interventional study also found that fertility education increased new medical consultations for fertility, especially when the educational subjects were married men [44]. Thus, interventions for increasing fertility awareness, especially among men, could help more people receive treatment earlier.

Gender inequality in the home provides another potential explanation for the lack of association between wives' education and help-seeking behavior. Domestic gender inequality may also explain the significant association between husbands' education and help seeking. Traditional gender roles are still entrenched in Japanese societies, as manifested by the unequal division of domestic work [45]. Greater gender equality is positively correlated with ART utilization in Europe [47]. It is therefore possible that delayed help seeking and lack of cooperation by husbands are caused by gender inequality within couples as well as gender disparities in fertility literacy.

Unemployment of wives was associated with 1.5 times the odds of medical help-seeking, compared to full-time employment. Women with paid work were generally less likely to seek medical help for fertility [46]. As more and more couples in developed countries choose to remain childless [47], those experiencing less desire for children will be unlikely to seek medical help. However, we should note that women continue to experience difficulties balancing work with fertility plans. Once people sought medical help, they often discontinued fertility treatment due to the difficulty in integrating therapeutic programs with their work [48]. In Japan, 8\% of working women quit their job due to fertility treatments [49]. Although our analyses were based on a cross-sectional survey and employment status was obtained at the time of the survey, employment would negatively affect seeking medical advice and receiving treatments. The low share of women in senior roles in Japan [50] suggests that it may be difficult for women to get permission to take time off work for infertility treatments, and then to return to their career track after pregnancy and childbirth. Furthermore, the wide gender gap in political empowerment [50] has delayed the development of legislation to correct these workplace disadvantages. As more women join the labor force, policies to increase public understanding of infertility and help employees balance work and fertility treatment are necessary.

To our knowledge, this is the first study to identify socioeconomic gaps in help-seeking for fertility problems in Japan. In the face of severe low fertility and increased infertility, the government has revised the public subsidy system for ART treatments many times [51] and recently decided to modify health insurance coverage for ART treatment starting in 2022 to reduce the financial burden for infertile patients [52]. With this coverage, more people who seek help will be able to receive advanced treatments. However, this policy change does not address those patients in Japan who are unlikely to seek help in 
the first place. Our study recommends future research and discussion to support people with fertility problems, including non-help-seekers.

Several limitations of this study should be acknowledged. First, the National Fertility Survey was a cross-sectional analysis, and thus we could obtain socioeconomic information at the time of the survey only. However, we confirmed similar associations between income and help-seeking behavior even among those with current fertility problems (Additional file 1: Table S2). Second, we could not fully account for the motivation to have a child, although desire to have a child was included in our multivariable models. As intentional childlessness is now widely accepted in developed countries [47], attitudes toward childbearing should be assessed in future research.

Third, the Married Couples Survey of the 15th National Fertility Survey evaluated legally married couples only. We were therefore unable to investigate attitudes to fertility across the full spectrum of the Japanese population. However, we believe our results were representative of national birth trends, because $98 \%$ of births in Japan are registered to legally married heterosexual couples [53]. A national survey on attitudes to fertility and children among non-heteronormative individuals and couples will be needed in the future.

Fourth, we performed the analyses without accounting for cluster sampling or stratification because data on stratification and sampling units were not available. Although this did not affect the point estimates [54, 55], the standard errors of the estimated odds ratio might have been affected: the lack of accounting for clustering could have led to an overestimate of the standard error, and the omission of stratification data could have led to an underestimate of the standard error [55]. Thus, confidence intervals in our analyses should be interpreted with caution. Finally, although the response rate to the National Fertility Survey was high (87.8\%), non-respondents may have introduced selection bias into our findings. However, the distribution of age, employment status and educational level across the respondents were almost equivalent to that of the national population $[31,56]$.

\section{Conclusions}

This study assessed the association between household income and the seeking of medical help for fertility problems using nationally representative survey data. Although Japan provides public funding for fertility treatments, we found that higher household income was associated with a higher probability of help-seeking among couples with fertility problems. Further in-depth studies to investigate related factors (e.g., financial, societal, cultural, and psychological) would be needed to inform future policy-making and improve the situations for those suffering from fertility problems.

Abbreviations

ART: Assisted reproductive technology; JPY: Japanese Yen; USD: US dollars; OR: Odds ratio; Cl: Confidence interval; aOR: Adjusted OR.

\section{Supplementary Information}

The online version contains supplementary material available at https://doi. org/10.1186/s12978-021-01212-w.

Additional file 1. Table S1. Sociodemographic characteristics of participants who had reported current fertility problems according to the status of medical help-seeking. Table S2. Logistic regression analysis for factors associated with medical help-seeking among participants who had reported current fertility problems.

\section{Acknowledgements}

We thank Ms. Jennifer Keane and Mr. Andrew Park of Oxford Editing for editing the article.

\section{Authors' contributions}

Al and EM conceived and designed the study. Al had access to all the data for this project and performed the statistical analysis. Al and EM wrote the first draft of the paper. Al, EM and YK contributed the acquisition of data. All authors contributed the interpretation of data and EM, SCJ, AY, KS, AK, HS, $\mathrm{Ol}$ and $\mathrm{YK}$ contributed to critical revision of the manuscript for important intellectual content. All authors approved the final version for publication. YK supervised the study. All authors read and approved the final manuscript.

\section{Funding}

This work was supported by the Health and Labour Sciences Research Grant (H30-Sukoyaka-Ippan-002) and the Japan Society for the Promotion of Science: KAKENHI (Grant Number 21K03193).

\section{Availability of data and materials}

The datasets generated or analyzed during the current study are not publicly available due to restrictions on data-sharing for the National Fertility Survey of Japan, managed by the National Institute of Population and Social Security Research, under the Japanese Ministry of Health, Labour and Welfare.

\section{Declarations}

Ethics approval and consent to participate

The study protocol was approved by the institutional review board of the Graduate School of Medicine at The University of Tokyo (approval no: $2019270 \mathrm{NI}$, approved on January 23, 2020) and by the ethics committee of Akita University Graduate School of Medicine (approval no: 2300, approved on September 20, 2019). The Ministry of Health, Labor and Welfare approved the secondary use of data from the National Fertility Survey, and informed consent was not required because of anonymous data.

\section{Consent for publication}

Not applicable.

\section{Competing interests}

The authors declare that they have no competing interests with respect to this research study and paper.

\section{Author details}

${ }^{1}$ Department of Public Health, Graduate School of Medicine, The University of Tokyo, 7-3-1 Hongo, Bunkyo-ku, Tokyo 113-0033, Japan. ${ }^{2}$ Department of Environmental Health Science and Public Health, Akita University Graduate School of Medicine, 1-1-1 Hondo, Akita, Akita 010-8543, Japan. ${ }^{3}$ Department of Obstetrics and Gynecology, Saitama Medical University, 38 Morohongo, 
Moroyama-machi, Iruma, Saitama 350-0495, Japan. ${ }^{4}$ Department of Pediatrics, Perinatal, and Maternal Medicine (Ibaraki), Graduate School, Tokyo Medical and Dental University, 1-5-45 Yushima, Bunkyo-ku, Tokyo 113-8510, Japan. ${ }^{5}$ Department of Obstetrics and Gynecology, Graduate School of Biomedical Sciences, Tokushima University, 3-18-15 Kuramoto-cho, Tokushima, Tokushima 770-8503, Japan. ${ }^{6}$ Umegaoka Women's Clinic, 1-33-3 Umegaoka, Setagaya-ku, Tokyo 154-0022, Japan. ${ }^{7}$ Department of Obstetrics and Gynecology, Akita University Graduate School of Medicine, 1-1-1 Hondo, Akita, Akita 010-8543, Japan.

Received: 15 March 2021 Accepted: 19 July 2021

Published online: 03 August 2021

\section{References}

1. Boivin J, Bunting L, Collins JA, Nygren KG. International estimates of infertility prevalence and treatment-seeking: potential need and demand for infertility medical care. Hum Reprod. 2007;22:1506-12. https://doi.org/ 10.1093/humrep/dem046.

2. Zegers-Hochschild F, Adamson GD, Dyer S, Racowsky C, De Mouzon J, Sokol R, et al. The international glossary on infertility and fertility care, 2017. Hum Reprod. 2017;32:1786-801.

3. Division of Reproductive Health, National Center for Chronic disease Prevention and Health Promotion. 2016 Assisted Reproductive Technology National Summary Report. https://www.cdc.gov/art/pdf/2016-report/ ART-2016-National-Summary-Report.pdf. Accessed 4 Mar 2021.

4. De Geyter C, Calhaz-Jorge C, Kupka MS, Wyns C, Mocanu E, Motrenko T, et al. ART in Europe, 2015: results generated from European registries by ESHRE†. Hum Reprod Open. 2020;2020:1-17.

5. Zegers-Hochschild F, Schwarze JE, Crosby JA, Musri C, Urbina MT. Assisted reproductive techniques in Latin America: The Latin American registry, 2016. JBRA Assist Reprod. 2019;23:255-67.

6. Kushnir VA, Barad DH, Albertini DF, Darmon SK, Gleicher N. Systematic review of worldwide trends in assisted reproductive technology 2004-2013. Reprod Biol Endocrinol. 2017;15:1-9. https://doi.org/10.1186/ s12958-016-0225-2.

7. Baird DT, Collins J, Egozcue J, Evers LH, Gianaroli L, Leridon H, et al. Fertility and ageing. Hum Reprod Update. 2005;11:261-76.

8. Connolly MP, Hoorens S, Chambers GM. The costs and consequences of assisted reproductive technology: an economic perspective. Hum Reprod Update. 2010;16:603-13. https://doi.org/10.1093/humupd/ dmq013.

9. Mills M, Rindfuss RR, McDonald P, te Velde E. Why do people postpone parenthood? Reasons and social policy incentives. Hum Reprod Update. 2011;17:848-60.

10. Moreau C, Bouyer J, Ducot B, Spira A, Slama R. When do involuntarily infertile couples choose to seek medical help? Fertil Steril. 2010;93:737-44.

11. Datta J, Palmer MJ, Tanton C, Gibson LJ, Jones KG, Macdowall W, et al. Prevalence of infertilityand help seeking among 15000 women and men. Hum Reprod. 2016;31:2108-18.

12. Smith JF, Eisenberg ML, Glidden D, Millstein SG, Cedars M, Walsh TJ, et al. Socioeconomic disparities in the use and success of fertility treatments: analysis of data from a prospective cohort in the United States. Fertil Steril. 2011;96:95-101. https://doi.org/10.1016/j.fertnstert.2011.04.054.

13. Farland LV, Collier A-RY, Correia KF, Grodstein F, Chavarro JE, Rich-Edwards $J$, et al. Who receives a medical evaluation for infertility in the United States? Fertil Steril. 2016;105:1274-80. https://doi.org/10.1016/j.fertnstert. 2015.12.132.

14. Farland LV, Missmer SA, Rich-Edwards J, Chavarro JE, Barbieri RL, Grodstein F. Use of fertility treatment modalities in a large United States cohort of professional women. Fertil Steril. 2014;101:1705-10. https://doi.org/10. 1016/j.fertnstert.2014.03.016.

15. Farley Ordovensky Staniec J, Webb NJ. Utilization of infertility services: how much does money matter? Health Serv Res. 2007;42:971-89. https:// doi.org/10.1111/j.1475-6773.2006.00640.x.

16. Chambers GM, Hoang VP, Illingworth PJ. Socioeconomic disparities in access to ART treatment and the differential impact of a policy that increased consumer costs. Hum Reprod. 2013;28:3111-7. https://doi.org/ 10.1093/humrep/det302.

17. Griesinger G, Diedrich K, Altgassen C. Stronger reduction of assisted reproduction technique treatment cycle numbers in economically weak geographical regions following the German healthcare modernization law in 2004. Hum Reprod. 2007;22:3027-30. https://doi.org/10.1093/ humrep/dem293.

18. Bitler MP, Schmidt L. Utilization of infertility treatments: the effects of insurance mandates. Demography. 2012;49:125-49. https://doi.org/10. 1007/s13524-011-0078-4.

19. Jain T, Harlow BL, Hornstein MD. Insurance coverage and outcomes of in vitro fertilization. N Engl J Med. 2002;347:661-6. https://doi.org/10. 1056/NEJMsa013491.

20. Ministry of Health, Labour and Welfare: Handbook of Health and Welfare Statistics. https://www.mhlw.go.jp/english/database/db-hh/1-2.html. Accessed 4 Mar 2021.

21. National Institute of Population and Social Security Research. Marriage and Childbirth in Japan Today (in Japanese). 2015. http://www.ipss.go.jp/ ps-doukou/j/doukou15/NFS15_reportALL.pdf. Accessed 4 Mar 2021.

22. Ikegami N, Yoo BK, Hashimoto H, Matsumoto M, Ogata H, Babazono A, et al. Japanese universal health coverage: evolution, achievements, and challenges. Lancet. 2011;378:1106-15. https://doi.org/10.1016/S01406736(11)60828-3.

23. Ministry of Health Labour and Welfare: Support for couples struggling with infertility (in Japanese). https://www.mhlw.go.jp/stf/seisakunitsuite/ bunya/0000047270.html. Accessed 4 Mar 2021.

24. Chambers GM, Hoang VP, Sullivan EA, Chapman MG, Ishihara O, ZegersHochschild F, et al. The impact of consumer affordability on access to assisted reproductive technologies and embryo transfer practices: an international analysis. Fertil Steril. 2014;101:191-198.e4. https://doi.org/10. 1016/j.fertnstert.2013.09.005.

25. Maeda E. Health and Labour Sciences Research Grants (H30-SukoyakaIppan-002) Summary Research Report (in Japanese). 2018. https://mhlwgrants.niph.go.jp/project/27041/1.

26. Chambers GM, Sullivan EA, Ishihara O, Chapman MG, Adamson GD. The economic impact of assisted reproductive technology: a review of selected developed countries. Fertil Steril. 2009;91:2281-94. https://doi. org/10.1016/J.FERTNSTERT.2009.04.029.

27. Niino Y, Okai T. A study of public support for patients being treated for infertility : the first report (in Japanese). Jpn J Matern Health. 2008;49:138-44.

28. National Institute of Population and Social Security Research. The 15th Japanese National Fertility. http://www.ipss.go.jp/ps-doukou/e/douko u15/Nfs15_gaiyoEng.html. Accessed 4 Mar 2021.

29. Ghaznavi C, Sakamoto H, Yoneoka D, Nomura S, Shibuya K, Ueda P. Trends in heterosexual inexperience among young adults in Japan: Analysis of national surveys, 1987-2015. BMC Public Health. 2019;19:355. https://doi. org/10.1186/s12889-019-6677-5.

30. Statistics Bureau Website/ What is a Densely Inhabited District? https:// www.stat.go.jp/english/data/chiri/did/1-1.html. Accessed 4 Mar 2021.

31. Statistics Bureau Website/ Final report of 2015 "POPULATION AND HOUSEHOLDS OF JAPAN." https://www.stat.go.jp/english/data/kokusei/ 2015/final en/final en.html. Accessed 4 Mar 2021.

32. Young R, Johnson DR. Imputing the Missing Y's: implications for Survey Producers and Survey Users.

33. Lee KJ, Roberts G, Doyle LW, Anderson PJ, Carlin JB. Multiple imputation for missing data in a longitudinal cohort study: a tutorial based on a detailed case study involving imputation of missing outcome data. Int J Soc Res Methodol. 2016;19:575-91. https://doi.org/10.1080/13645579. 2015.1126486.

34. Zhou Z, Zheng D, Wu H, Li R, Xu S, Kang Y, et al. Epidemiology of infertility in China: a population-based study. BJOG An Int J Obstet Gynaecol. 2018;125:432-41. https://doi.org/10.1111/1471-0528.14966.

35. da Silva SG, Bertoldi AD, da Silveira MF, Domingues MR, Evenson KR, dos Santos IS. Assisted reproductive technology: prevalence and associated factors in Southern Brazil. Rev Saude Publica. 2019;53:13.

36. Bureau of Social Welfare and Public Health, Tokyo Metropolitan Government: Summary of the Subsidy Program for Infertility Testing (in Japanese). https://www.fukushihoken.metro.tokyo.lg.jp/kodomo/kosodate/ josei/funinkensa/gaiyou.html. Accessed 4 Mar 2021. 
37. Minato City Specified Fertility Treatment Subsidy Program Guide FY2020. https://www.city.minato.tokyo.jp/chiikihoken/kenko/ninshin/ninshin/ kibo/h23jose.html. Accessed 28 Jul 2021.

38. About happiness hump stork business Akita prefecture specific fertility treatment expense subsidy business/Specified fertility treatment cost subsidy business (in Japanese). https://www.pref.akita.lg.jp/pages/archi ve/862. Accessed 4 Mar 2021.

39. Kessler LM, Craig BM, Plosker SM, Reed DR, Quinn GP. Infertility evaluation and treatment among women in the United States. Fertil Steril. 2013;100:1025-32. https://doi.org/10.1016/j.fertnstert.2013.05.040.

40. Maeda E, Sugimori H, Nakamura F, Kobayashi Y, Green J, Suka M, et al. A cross sectional study on fertility knowledge in Japan, measured with the Japanese version of Cardiff Fertility Knowledge Scale (CFKS-J). Reprod Health. 2015;12:10. https://doi.org/10.1186/1742-4755-12-10.

41. Bunting $L$, Boivin J. Decision-making about seeking medical advice in an internet sample of women trying to get pregnant. Hum Reprod. 2007;22:1662-8. https://doi.org/10.1093/humrep/dem057.

42. Fulford B, Bunting L, Tsibulsky I, Boivin J. The role of knowledge and perceived susceptibility in intentions to optimize fertility: findings from the International Fertility Decision-Making Study (IFDMS). Hum Reprod. 2013;28:3253-62. https://doi.org/10.1093/humrep/det373.

43. Hiroshima Prefecture|Result of Fertility Survey (in Japanese). https:// www.pref.hiroshima.Ig.jp/soshiki/248/ninnkatudaityousakekka.html. Accessed 27 Jun 2021

44. Maeda E, Boivin J, Toyokawa S, Murata K, Saito H. Two-year follow-up of a randomized controlled trial: knowledge and reproductive outcome after online fertility education. Hum Reprod. 2018;33:2035-42. https://doi.org/ 10.1093/humrep/dey293.

45. OECD.Stat. Employment: Time spent in paid and unpaid work, by sex 2019. https://stats.oecd.org/index.aspx?queryid=54757. Accessed $1 \mathrm{Jul}$ 2021.

46. Olsen J, Basso O, Spinelli A, Küppers-Chinnow M, Juul S, Karmaus W, et al. Correlates of care seeking for infertility treatment in Europe: implications for health services and research. Eur J Public Health. 1998:8:15-20.

47. Miettinen A, Rotkirch A, Szalma I, Donno A, Tanturri M-L. Increasing childlessness in Europe: time trends and country differences. 2015. http:// www.familiesandsocieties.eu/wp-content/uploads/2015/03/WP33Mietti nenEtAl2015.pdf.
48. Gameiro S, Boivin J, Peronace L, Verhaak CM. Why do patients discontinue fertility treatment? A systematic review of reasons and predictors of discontinuation in fertility treatment. Hum Reprod Update. 2012;18:652-69. https://doi.org/10.1093/humupd/dms031.

49. Ichikawa T, Ota I, Kuwabara Y, Tsushima R, Hamatani T, Hiraike O, et al. Infertility treatment hinders the careers of working women in Japan. J Obstet Gynaecol Res. 2020;46:1940-50. https://doi.org/10.1111/jog. 14387.

50. Global Gender Gap Report 2021. 2021. https://jp.weforum.org/reports/ global-gender-gap-report-2021. Accessed 27 Jun 2021.

51. Maeda E, Ishihara O, Saito H, Kuwahara A, Toyokawa S, Kobayashi Y. Agespecific cost and public funding of a live birth following assisted reproductive treatment in Japan. J Obstet Gynaecol Res. 2014;40:1338-44. https://doi.org/10.1111/jog.12337.

52. Japan health ministry to seek more funds for fertility treatment $\mid$ The Japan Times. https://www.japantimes.co.jp/news/2020/09/25/national/ japan-health-ministry-infertility/. Accessed 4 Mar 2021.

53. Ministry of Health, Labour, and Welfare. Vital Statistics (in Japanese). 2015. https://www.e-stat.go.jp/stat-search/files?page=1\&layout=datal ist\&toukei $=00450011 \&$ tstat $=000001028897 \&$ cycle $=7 \&$ year $=20150 \&$ month $=0 \&$ tclass $1=000001053058 \&$ tclass $2=000001053061 \&$ tclass $3=$ $000001053064 \& t c l a s s 4 \mathrm{val}=0$. Accessed $1 \mathrm{Jul} 2021$.

54. Bell BA, Onwuegbuzie AJ, Ferron JM, Jiao QG, Hibbard ST, Kromrey JD. Use of design effects and sample weights in complex health survey data: a review of published articles using data from 3 commonly used adolescent health surveys. Am J Public Health. 2012;102:1399-405.

55. StataCorp LP. Stata Survey Data Reference Manual (Release 16). 2019. https://www.stata.com/manuals/svy.pdf. Accessed 4 Mar 2021.

56. Statistics Bureau Website/ POPULATION AND HOUSEHOLDS OF JAPAN 2010. https://www.stat.go.jp/english/data/kokusei/2010/poj/mokuji.html. Accessed 4 Mar 2021

\section{Publisher's Note}

Springer Nature remains neutral with regard to jurisdictional claims in published maps and institutional affiliations.
Ready to submit your research? Choose BMC and benefit from:

- fast, convenient online submission

- thorough peer review by experienced researchers in your field

- rapid publication on acceptance

- support for research data, including large and complex data types

- gold Open Access which fosters wider collaboration and increased citations

- maximum visibility for your research: over $100 \mathrm{M}$ website views per year

At BMC, research is always in progress.

Learn more biomedcentral.com/submissions 